\title{
Search for nearby stars among proper motion stars selected by optical-to-infrared photometry
}

\section{Two late $\mathrm{M}$ dwarfs within $10 \mathrm{pc}^{\star}$}

\author{
M. J. McCaughrean, R.-D. Scholz, and N. Lodieu
}

\begin{abstract}
Astrophysikalisches Institut Potsdam, An der Sternwarte 16, 14482 Potsdam, Germany
e-mail: rdscholz@aip.de; nlodieu@aip.de
\end{abstract}

Received 7 May 2002 / Accepted 17 June 2002

\begin{abstract}
We have identified two late M dwarfs within $10 \mathrm{pc}$ of the Sun, by cross-correlating the Luyten NLTT catalogue of stars with proper motions larger than $0.18 \mathrm{arcsec} / \mathrm{yr}$, with objects lacking optical identification in the 2MASS data base. The 2MASS photometry was then combined with improved optical photometry obtained from the SuperCOSMOS Sky Surveys. The two objects (LP 775-31 and LP 655-48) have extremely red optical-to-infrared colours $\left(\left(R-K_{\mathrm{s}}\right) \sim 7^{\mathrm{m}}\right)$ and very bright infrared magnitudes $\left(K_{\mathrm{s}}<10^{\mathrm{m}}\right)$ : follow-up optical spectroscopy with the ESO 3.6-m telescope gave spectral types of M 8.0 and M 7.5 dwarfs, respectively. Comparison of their near-infrared magnitudes with the absolute magnitudes of known M 8 and M7.5 dwarfs with measured trigonometric parallaxes yields spectroscopic distance estimates of $6.4 \pm 1.4 \mathrm{pc}$ and $8.0 \pm 1.6 \mathrm{pc}$ for LP 775- 31 and LP 655-48, respectively. In contrast, Cruz \& Reid (2002) recently determined spectral types of M 6 for both objects, and commensurately larger distances of $11.3 \pm 1.3 \mathrm{pc}$ and $15.3 \pm 2.6 \mathrm{pc}$. LP $655-48$ is also a bright X-ray source (1RXS J044022.8-053020). With only a few late M dwarfs previously known within $10 \mathrm{pc}$, these two objects represent an important addition to the census of the Solar neighbourhood.
\end{abstract}

Key words. astrometry and celestial mechanics: astrometry - astronomical data base: surveys - stars: late-type stars: low mass, brown dwarfs

\section{Introduction}

The census of the solar neighbourhood remains very incomplete. From the statistics of the Catalogue of Nearby Stars (Gliese \& Jahreiß 1991), one can infer that about $30 \%$ of all stars (and probably an even larger fraction of the substellar brown dwarfs) within $10 \mathrm{pc}$ are as yet undetected. These sources are important however, as the detailed observation of very nearby stars and brown dwarfs is one of the main starting points for investigations of the star formation process, the stellar and substellar luminosity function, and the initial mass function. Furthermore, future missions for the detection of extrasolar planets (e.g., SIM, TPF, Darwin) will concentrate on very nearby stars in order to reveal Earthlike planets. As a result, several major efforts are underway to provide a more detailed census of the solar neighbourhood, including the RECONS and NStars projects, out to 10 and $25 \mathrm{pc}$, respectively (www.chara.gsu.edu/RECONS and

\footnotetext{
Send offprint requests to: M. J. McCaughrean, e-mail: mjm@aip.de

* Based on observations collected with the ESO 3.6-m/EFOSC2 at the European Southern Observatory, La Silla, Chile (ESO programme 68.C-0664).
}

nstars . arc . nasa.gov). Recent discoveries in the immediate solar neighbourhood $(<15 \mathrm{pc})$ include early and mid $\mathrm{M}$ dwarfs (Jahreiß et al. 2001; Scholz et al. 2001; Phan-Bao et al. 2001; Scholz et al. 2002a; Reid \& Cruz 2002; Reid et al. 2002a; Cruz \& Reid 2002; Reylé et al. 2002), late M and L dwarfs (Gizis et al. 2000; Kirkpatrick et al. 2000; Reid et al. 2000; Delfosse et al. 2001; Reid \& Cruz 2002; Reid et al. 2002a; Cruz \& Reid 2002; Lodieu et al. 2002), and cool white dwarfs (Reid et al. 2001; Scholz et al. 2002b).

All of these objects show a proper motion larger than the NLTT (New Luyten Two Tenths catalogue; Luyten 1979-1980) limit of $0.18 \mathrm{arcsec} / \mathrm{yr}$, and indeed, many of the early and mid $\mathrm{M}$ dwarfs were selected from the NLTT. More than 20 years after its publication, the NLTT is still an important source in the search for nearby stars and by far not yet fully exploited. North of $\delta=-33^{\circ}$, the NLTT catalogue is based on Palomar Schmidt plates and has a limiting magnitude of $m_{\mathrm{pg}} \sim 20.5$ (see, e.g., Reid \& Cruz 2002). South of $\delta=-33^{\circ}$, the limiting magnitude of the NLTT is only $m_{\mathrm{pg}} \sim 15 \mathrm{~m} .5$, and thus new high proper motion surveys have been started using UK Schmidt Telescope (UKST) and ESO Schmidt plates (Scholz et al. 2000; Ruiz et al. 2001). 
Many recent additions to the census of late $\mathrm{M}$ and early $\mathrm{L}$ dwarfs within $15 \mathrm{pc}$ were found in the near-infrared sky surveys 2MASS (Gizis et al. 2000; Kirkpatrick et al. 2000; Reid et al. 2000) and DENIS (Delfosse et al. 2001). Nevertheless, all these objects could in principle have been selected directly from the NLTT and/or modern Schmidt plate measurements, and in fact, all of them were subsequently identified as high proper motion objects in the NLTT or from Schmidt plates.

With the recent discovery of three nearby L dwarfs (one with a spectroscopic distance estimate of less than $15 \mathrm{pc}$ ) in the southern sky (Lodieu et al. 2002), we have demonstrated that high proper motion surveys can successfully contribute to the completion of the census of the solar neighbourhood at the stellar/substellar boundary. Furthermore, the combination of old and new proper motion catalogues with modern nearinfrared sky surveys is a very effective tool in the search for nearby red dwarfs. The NLTT and 2MASS have been used to discover an M 6.5 dwarf at $\sim 6 \mathrm{pc}$ (Scholz et al. 2001), and five new sources were identified within $15 \mathrm{pc}$ (and one closer than $10 \mathrm{pc}$ ) from a new proper motion survey combined with DENIS data (Reylé et al. 2002). Most recently, Reid \& Cruz (2002), Reid et al. (2002a), and Cruz \& Reid (2002) have reported the discovery of many additions to the immediate solar neighbourhood from NLTT stars identified in the 2MASS, including five objects within $10 \mathrm{pc}$,

In this paper, we describe the discovery of two very nearby $(<10 \mathrm{pc})$ late $\mathrm{M}$ dwarfs in the southern sky, also found in a combined search of the NLTT and 2MASS. The same two objects were also recently identified by Cruz \& Reid (2002) with different results, as described below.

\section{Re-identification and photometry of NLTT stars}

In order to identify the high proper motion stars of the NLTT catalogue (Luyten 1979-1980) in the 2MASS data base, a reidentification of the objects in the optical is first necessary. As recently shown by Gould \& Salim (2002), Luyten measured positions for the majority of the stars in his catalogue to 1 arcsec accuracy, but recorded the results to an accuracy of only $1 \mathrm{~s}$ of time in right ascension and 6 arcsec in declination. In addition, there is a long tail of positional errors ranging from tens to hundreds of arcsec. Thus, re-identification requires a visual inspection of multi-epoch data obtained from scans of photographic Schmidt plates.

Also, as the stars are re-identified, improved photographic photometry can be obtained to compensate for the relatively low photometric accuracy of the NLTT catalogue (Scholz et al. 2000; Gould \& Salim 2002; Reid \& Cruz 2002). In the northern sky, the USNO A2.0 catalogue (Monet et al. 1998) can be used, but south of $\delta=-20^{\circ}$, most NLTT stars are not included, due to the epoch difference between the Schmidt plates and the object matching radius used in the construction of the catalogue.

Thus here we have used the SuperCOSMOS Sky Survey (hereafter SSS: Hambly et al. 2001a, 2001b, 2001c), a recent scan of the entire southern sky made from UKST and ESO Schmidt plates in three passbands $\left(B_{\mathrm{J}}, R\right.$, and $\left.I\right)$. The SSS data are available in the form of images which can be used for the reidentification of NLTT stars, and object catalogues, including accurate photographic photometry from each of the plates. The epoch differences between the plates vary from field to field, with typically a maximum of 15-20 years between the UKST $B_{\mathrm{J}}$ and $R$ plates. The quality of and easy access to the SSS data make it well suited to the re-identification and photometry of NLTT stars, and we have also used these data to conduct a new high proper motion survey which has led to the discovery of a new wide pair of cool white dwarfs in the solar neighbourhood (Scholz et al. 2002b) and three nearby L dwarfs (Lodieu et al. 2002).

\section{Selecting objects from the 2MASS}

To yield a list of candidate nearby red dwarfs in the southern sky, we first searched the 2MASS for bright $\left(K_{\mathrm{S}}<11^{\mathrm{m}}\right)$ southern stars without optical counterparts. As the USNO A2.0 catalogue is used to provide optical data for 2MASS stars, the large epoch difference between the A2.0 and 2MASS observations ensures that high proper motion stars usually end up without an optical counterpart in the 2MASS catalogue.

The resulting $>10^{6}$ 2MASS sources without optical counterparts were then cross-correlated with the NLTT catalogue, using a search radius of 60 arcsec. In practice, most matches were found in the region $-33^{\circ}<\delta<0^{\circ}$ due to the limited NLTT sensitivity south of that: of this region, roughly $90 \%$ is covered in the publicly-available 2MASS database. For any objects thus identified, a very large optical-to-infrared colour index of $\left(m_{\mathrm{r}}-K_{\mathrm{s}}\right)>6^{\mathrm{m}}$ was used as a further selection criterion, where $m_{\mathrm{r}}$ is the rough red magnitude estimate in the NLTT catalogue.

Only $\sim 170$ matches remained after this selection, and these were then re-identified and checked using SSS images, and $B_{\mathrm{J}}$, $R$, and $I$ photometry was obtained from the SSS catalogues. The final list of candidates consisted of just 9 NLTT entries with $\left(R-K_{\mathrm{s}}\right)>6.5$. Six of these were known M 7-M 9.5 dwarfs and one object was not visible during our spectroscopic followup observations. The two remaining candidates, LP 775-31 and LP 655-48, were among the brightest 2MASS sources in the sample of 9 objects. Other sources with comparable brightness $\left(K_{\mathrm{S}} \sim 9.5\right)$ include the known brown dwarf LP 944- 20 (Tinney 1996, 1998) and the recently discovered M9 dwarf DENISP J104814.7-395606.1 (Delfosse et al. 2001).

The full astrometric and photometric data for LP 775-31 and LP655-48 are given in Table 1. It is worth noting that LP 655-48 is also identified with a bright X-ray source (1RXS J044022.8-053020; Voges et al. 1999).

\section{Optical classification spectroscopy}

Optical classification spectroscopy was obtained for the two candidate nearby sources using EFOSC2 mounted on the ESO 3.6-m telescope on La Silla on 22 November 2001. The conditions were photometric and the seeing $\sim 0.6-0.8 \operatorname{arcsec} F W H M$. The spectrograph uses a $2048 \times 2048$ pixel Loral/Lesser CCD with a pixel size of $0.157 \mathrm{arcsec}$, and has a useable fieldof-view of $5.2 \times 5.2$ arcmin. A 1 arcsec slit was used with Grism 12 covering 6000-10300 $\AA$ at $R \sim 600$. Single exposures of $240 \mathrm{~s}$ were obtained for each of the two candidates, and 
Table 1. Astrometry and photometry from the SSS and 2MASS. The proper motion for each object is based on five positions: the $B_{\mathrm{J}}, R$, and $I$ plates measured within the SSS, the 2MASS position, and the USNO A2.0 early epoch position. The coordinates are given for the most recent epoch, which is 2MASS for LP 775- 31 and the SSS I plate for LP 655- 48.

\begin{tabular}{|c|c|c|c|c|c|c|c|c|c|c|}
\hline $\begin{array}{c}\text { Name } \\
\text { LP }\end{array}$ & $\begin{array}{c}\alpha, \delta \\
(\mathrm{J} 2000)\end{array}$ & Epoch & \multicolumn{2}{|c|}{$\mathrm{mas} / \mathrm{yr}$} & $B_{\mathrm{J}}$ & $(\mathrm{SSS})$ & $I$ & \multicolumn{2}{|c|}{ (2MASS) } & $K_{\mathrm{s}}$ \\
\hline $775-31$ & $043516.14-160657.5$ & 1998.90 & $+160 \pm 4$ & $+305 \pm 4$ & 18.85 & 16.35 & 12.36 & 10.40 & 9.78 & 9.34 \\
\hline $655-48$ & $044023.33-053007.9$ & 2001.80 & $+335 \pm 2$ & $+131 \pm 2$ & 18.85 & 16.50 & 13.17 & 10.68 & 9.99 & 9.56 \\
\hline
\end{tabular}

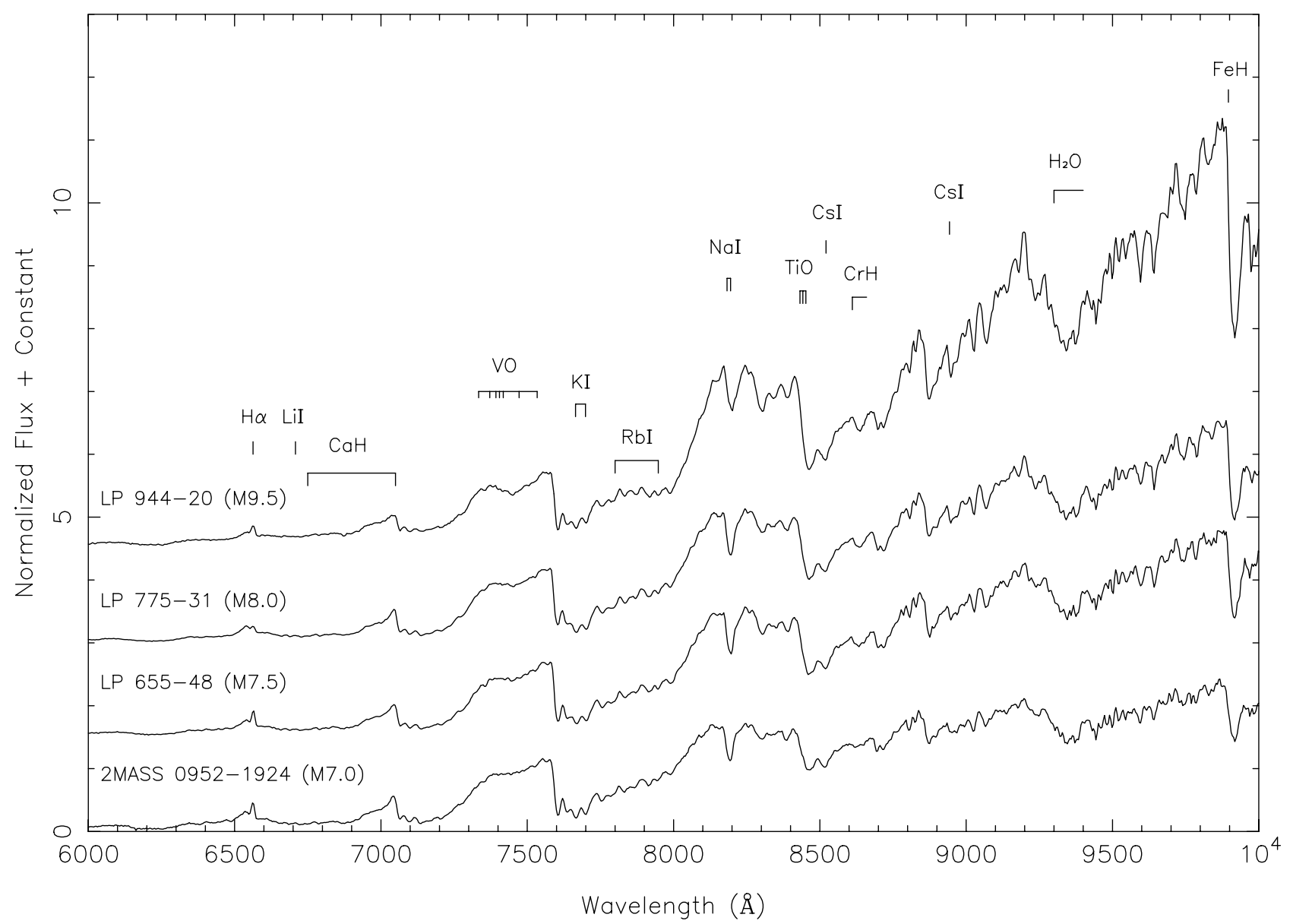

Fig. 1. ESO 3.6-m/EFOSC2 spectra of LP 775- 31 and LP 655- 48, compared with those of LP944- 20, a known M 9.5 brown dwarf (Tinney 1996, 1998; Reid et al. 2002b), and the M7.0 dwarf 2MASS 0952219-192431 (Gizis et al. 2000). An arbitrary constant has been used to separate the spectra. The location of features typical of late-type stars are labelled, including metal oxide and hydride absorption bands, atomic absorption lines, lithium absorption, and $\mathrm{H} \alpha$ emission.

of $200 \mathrm{~s}$ for each of two comparison late M dwarfs (LP 944- 20 and 2MASS 0952219-192431).

Data reduction was standard, including subtraction of an averaged dark frame and division by an internal quartz flat field to remove fringing above $8000 \AA$. Wavelength calibration was made using $\mathrm{He}$ and Ar lines over the whole wavelength range, and flux calibration was achieved using an averaged sensitivity function determined from several exposures of the spectrophotometric standard stars EG21 and LTT1788 taken during the night.

After extraction of the spectra, the PC3 index defined by Martín et al. (1999) was used to determine spectral types of M 6.5, M 7.5, M 8.0, and M 9.5 for 2MASS 0952219-192431, LP 655-48, LP 775-31, and LP 944-20, respectively (see
Fig. 1). Spectral types determined using the classification scheme of Kirkpatrick et al. (1999) were consistent to within the half subclass errors. The spectral types found for the comparison objects are in good agreement with previously published values: the M9.5 determined for LP944- 20 is consistent with the recent value given by Reid et al. (2002b), while Tinney (1996) gave it as M9 on its discovery.

Thus for the two new sources, LP 655-48 and LP 775-31, we adopt spectral types M 7.5 and M 8.0, respectively. We note that Cruz \& Reid (2002) gave M 6 for both stars, but the direct spectroscopic evidence presented here strongly favours the later types we have adopted. 


\section{Determination of spectroscopic distances}

The data of Reid et al. (2002b) yield trigonometric distances and absolute $M_{J}, M_{H}, M_{K_{\mathrm{s}}}$ magnitudes for three M 8 and four M7.5 dwarfs with 2MASS measurements. The abbreviated names of these objects are 2M0052-27, 2M0320+18, $2 \mathrm{M} 1242+29$ (M8), and 2M1504-23, 2M1524+29, 2M1527+41, 2M1550+30 (M 7.5). Their mean absolute magnitudes $M_{J}, M_{H}$, and $M_{K_{\mathrm{s}}}$ are $11^{\mathrm{m}} .45,10^{\mathrm{m}} .76,10^{\mathrm{m}} .28$ for the M 8 sources, and 11 . $11,10^{\mathrm{m}} .48,10^{\mathrm{m}} 07$ for the M 7.5 sources.

Using these reference data, we can estimate the distances to the new sources. For LP 775-31, we obtain $d_{J}=6.2 \mathrm{pc}$, $d_{H}=6.4 \mathrm{pc}$, and $d_{K_{\mathrm{s}}}=6.5 \mathrm{pc}$, and for LP 655-48, we obtain $d_{J}=8.2 \mathrm{pc}, d_{H}=8.0 \mathrm{pc}$, and $d_{K_{\mathrm{s}}}=7.9 \mathrm{pc}$. Making a conservative assumption of \pm 0.4 accuracy in the absolute magnitudes, we therefore estimate spectroscopic distances of $6.4 \pm 1.4 \mathrm{pc}$ for LP 775- 31 and $8.0 \pm 1.6 \mathrm{pc}$ for LP 655-48. It is worth noting that there is a significant possibility $(\sim 20 \%)$ of either object being a binary system (Close et al. 2002), in which case the distance given here would be an underestimate: in the worst case of an equal mass binary, the true distance would be $\sim 1.4$ times larger.

\section{Conclusions}

By combining data from the NLTT, 2MASS, and SSS, we have discovered two very nearby late M dwarfs: LP 775-31 has a spectral type of M 8 and lies at a distance of $6.4 \pm 1.4 \mathrm{pc}$, while LP 655-48 has a spectral type of M 7.5 and lies at $8.0 \pm 1.6 \mathrm{pc}$. Our spectroscopic distance estimates are roughly half those recently published by Cruz \& Reid (2002), namely $11.3 \pm 1.3$ pc and $15.3 \pm 2.6 \mathrm{pc}$, and need to be confirmed by checking the objects for a possible binary nature and by determining trigonometric parallaxes. If confirmed, they would be among the nearest stars to the Sun and new benchmark M 8 and M 7.5 dwarfs, allowing detailed follow-up investigations.

Acknowledgements. We have made use of data products from the SuperCOSMOS Sky Surveys at the Wide-Field Astronomy Unit of the Institute for Astronomy, University of Edinburgh, and from the Two Micron All Sky Survey, a joint project of the University of Massachusetts and IPAC, funded by NASA and the NSF. The authors would like to thank ESO 3.6-m support team for useful discussions, Nigel Hambly for advice on the use of SSS data, and Darja Golikowa and Kai Schmitz for their help with the re-identification of NLTT stars in DSS and SSS images and with the cross-correlation of USNO A2.0, SSS, and 2MASS data. NL and MJM thank the EC RTN "The Formation and Evolution of Young Stellar Clusters" (HPRN-CT-2000-00155).

\section{References}

Close, L. M., Siegler, N., Potter, D., Brandner, W., \& Liebert, J. 2002, ApJ, 567, L53

Cruz, K. L., \& Reid, I. N. 2002, AJ, 123, 2828

Delfosse, X., Forveille, T., Martín, E. L., et al. 2001, A\&A, 366, L13

Gizis, J. E., Monet, D. G., Reid, I. N., et al. 2000, AJ, 120, 1085

Gould, A., \& Salim, S. 2002, ApJ, in press [astro-ph/0204217]

Hambly, N. C., Davenhall, A. C., Irwin, M. J., \& MacGillivray, H. T. 2001c, MNRAS, 326, 1315

Hambly, N. C., Irwin, M. J., \& MacGillivray, H. T. 2001b, MNRAS, 326, 1295

Hambly, N. C., MacGillivray, H. T., Read, M. A., et al. 2001a, MNRAS, 326, 1279

Gliese, W., \& Jahreiß, H. 1991, Preliminary Version of the Third Catalogue of Nearby Stars, computer-readable version on ADC Selected Astronomical Catalogs vol. 1 - CD-ROM

Jahreiß, H., Scholz, R.-D., Meusinger, H., \& Lehmann I. 2001, A\&A, 370, 967

Kirkpatrick, J. D., Reid, I. N., Liebert, J., et al. 1999, ApJ, 519, 802

Kirkpatrick, J. D., Reid, I. N., Liebert, J., et al. 2000, AJ, 120, 447

Lodieu, N., Scholz, R.-D., \& McCaughrean, M. J. 2002, A\&A, in press

Luyten, W. J. 1979-1980, New Luyten Catalogue of Stars with Proper Motions Larger than Two Tenths of an Arcsecond, University of Minnesota, Minneapolis, computer-readable version on ADC Selected Astronomical Catalogs vol. 1 - CD-ROM

Martín, E. L., Delfosse, X., Basri, G., et al. 1999, AJ, 118, 2466

Monet, D., Bird, A., Canzian, B., et al. 1998, USNO-A V2.0, A Catalog of Astrometric Standards, U.S. Naval Observatory Flagstaff Station (USNOFS) and Universities Space Research Association (USRA) stationed at USNOFS

Phan-Bao, N., Guibert, J., Crifo, F., et al. 2001, A\&A, 380, 590

Reid, I. N., \& Cruz, K. L. 2002, AJ, 123, 2806

Reid, I. N., Kilkenny, D., \& Cruz, K. L. 2002a, AJ, 123, 2822

Reid, I. N., Kirkpatrick, J. D., Gizis, J. E., et al. 2000, AJ, 119, 369

Reid, I. N., Kirkpatrick, J. D., Liebert, J., et al. 2002b, AJ, in press [astro-ph/0204285]

Reid, I. N., Liebert, J., \& Schmidt, G. D. 2001, ApJ, 550, L61

Reylé, C., Robin, A. C., Scholz, R.-D., \& Irwin, M. J. 2002, A\&A, submitted

Ruiz, M. T., Wischnjewsky, M., Rojo, P. M., \& Gonzalez, L. E. 2001, ApJSS, 133, 119

Scholz, R.-D., Ibata, R., Irwin, M. J., et al. 2002a, MNRAS, 329, 109

Scholz, R.-D., Irwin, M., Ibata, R., et al. 2000, A\&A, 353, 958

Scholz, R.-D., Meusinger, H., \& Jahreiß, H. 2001, A\&A, 374, L12

Scholz, R.-D., Szokoly, G. P., Andersen, M., et al. 2002b, ApJ, 565, 539

Tinney, C. G. 1996, MNRAS, 281, 644

Tinney, C. G. 1998, MNRAS, 296, L42

Voges, W., Aschenbach, B., Boller, Th., et al. 1999, A\&A, 349, 389 